\title{
DAMPING IN CFRP STRUCTURES: MODELLING AND COMPARISON OF TECHNOLOGICAL SOLUTIONS USING ELASTOMER
}

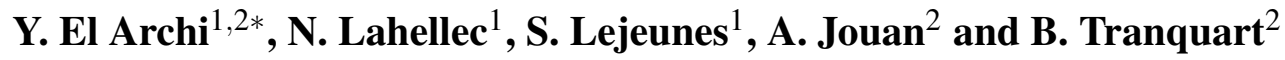 \\ ${ }^{1}$ LMA, UMR 7031, Aix-Marseille Univ, CNRS, Centrale Marseille, Marseille, France \\ ${ }^{2}$ Safran Composites, Itteville, France \\ *elarchi@1ma.cnrs-mrs.fr
}

In aeronautics, the latest generations of turbojets have inlet fan blades made of 3D-woven carbon fibre composite. Damping of rotating structures, such as blades, is of major industrial concern for controlling vibratory instabilities, like in-flight flutter for example, which can lead to the degradation of these blades. In order to overcome this difficulty and because CFRP materials are known to have limited damping properties, it seems necessary to implement innovative solutions to improve such a structure's damping.

Here, we study three damping technologies, based on the introduction of a dissipative material (elastomer) in the structure: The first technology consists in the bonding of an elastomeric layer at the scale of the blade structure which is also known as the viscoelastic patch (see [1]). Here the solution studied is a particular case of this technology where the elastomer layer lies within the laminate. The second technology consists in the introduction of the elastomer at the microstructure scale by adding some elastomeric fibers in the carbon fabric (see for example [2]). In the last one, still at the microstructure scale, all the carbon fibers are coated with a thin layer of elastomer ([3]).

In the present study, some multiscale simulations are done to demonstrate the ability of these techniques to damp the first eigenfrequency of a cantilever beam, also known as Oberst beam tests ([4]). The constitutive materials (epoxy matrix and elastomer) are modelled in the framework of linear viscoelasticity, the plies behaviour are obtained by numerical homogenization and the laminate response is obtained by finite element method analysis ( $\ll$ Steady-State Dynamics $\gg$ procedure, ABAQUS V6.14).

The results show that for a quasi-isotropic laminate stacking sequence, $[-45,90,45,0]_{s}$, the "coated" and "co-fabric" technologies succeed in damping the first eigenmode but the damping ratio obtained with both techniques are lower than those obtained with the patch method (all the results are obtained with the same amount of added elastomer).

\section{References}

[1] M. Martinez-Agirre, S. Illescas and M.J. Elejabarrieta, 2014, "Characterisation and Modelling of Prestrained Viscoelastic Films", International Journal of Adhesion and Adhesives, Vol. 50, pp. 183-190.

[2] A. Martone, M. Giordano, V. Antonucci and M. Zarrelli, Enhancing damping features of advanced polymer composites by micromechanical hybridization, Composites Part A, 2011.

[3] Fei Gao, Hang Song, Xiaowen Qiu, Fang He, Chunsheng Shi, Naiqin Zhao, Chuannian He, The Preparation and Properties of Novel Structural Damping Composites Reinforced by Nitrile Rubber Coated 3-D Braided Carbon Fibers, Polymer Composites 2019.

[4] Nashif, A. D., Jones, D. I., and Henderson, J. P. (1985). Vibration damping. John Wiley \& Sons. 